\title{
APPROXIMATE ZEROS OF QUADRATICALLY CONVERGENT ALGORITHMS
}

\author{
PENGYUAN CHEN
}

\begin{abstract}
Smale's condition for a point to be an approximate zero of a function for Newton's method is extended to the general quadratically convergent iterative algorithm. It is shown in which way the bound in the condition is affected by the characteristics of the algorithm. This puts the original condition of Smale for Newton's method in a more general perspective. The results are also discussed in the light of numerical evidence.
\end{abstract}

\section{INTRODUCTION}

When an iterative zero-seeking algorithm has a local quadratic convergence property, the iteration, if it ever converges, will eventually display the so-called precision-doubling phenomenon at each step, i.e., the precision of approximation is asymptotically doubled at each iteration. The traditional approach to the problem is largely based on asymptotic analysis, which often involves large unknown constants in the estimation and only provides a qualitative description of the convergence property. It is of practical use to have some kind of quantitative criterion for predicting the immediate appearance of such fast convergence. This kind of knowledge is also useful in the construction of more efficient algorithms and in the analysis of their efficiency (cf. $[3,4,5,6,7,9]$ ).

A point where fast convergence to a zero starts immediately under iteration is called an approximate zero. Several versions of this notion exist for the wellknown Newton's method and its higher-order generalizations (cf. [4, 5, 6, 7, 8]). The definition in [8], which is solely in terms of the convergence property and reflects the order of convergence, seems most appealing. In that paper, a point $z_{0}$ is called an approximate zero of $f$ for Newton's method if the Newton sequence $\left\{z_{k}\right\}$, where $z_{k}=z_{k-1}-f\left(z_{k-1}\right) / f^{\prime}\left(z_{k-1}\right)$ for $k=1,2, \ldots$, starting with $z_{0}$ satisfies the following fast convergence condition:

$$
\left|z_{k+1}-z_{k}\right| \leq\left(\frac{1}{2}\right)^{2^{k}-1}\left|z_{1}-z_{0}\right|, \quad k=0,1,2, \ldots .
$$

Under this definition, Smale [8] proved the following theorem that gives a

Received by the editor February 11, 1992 and, in revised form, January 4, 1993 and August 19, 1993.

1991 Mathematics Subject Classification. Primary 65H05; Secondary 65E05, 65 Y20.

This paper is a modified and extended version of part 4 of the author's thesis at Northwestern University. The author would like to thank the editor and the anonymous referee for suggesting some improvements. 
sufficient condition for a point to be an approximate zero based on estimates from data at that point.

Theorem (Smale). There is a constant $\alpha_{0} \approx 0.130707$ such that $z_{0}$ is an approximate zero of $f$ for Newton's method if $\gamma_{f}\left(z_{0}\right)\left|f\left(z_{0}\right)\right| /\left|f^{\prime}\left(z_{0}\right)\right|<\alpha_{0}$, where

$$
\gamma_{f}(z)=\sup _{k>1}\left|\frac{f^{(k)}(z)}{k ! f^{\prime}(z)}\right|^{\frac{1}{k-1}} .
$$

This work was later extended by Curry [1] and Kim [3] to some higher-order iterative algorithms including Euler's method and Halley's method. Although their definitions of an approximate zero are not exactly the same as that of Smale [8], the conditions they obtained are essentially the same, except for a different constant $\alpha_{0}$.

Our paper is in the same spirit as those works. We deal with the general Newton-like quadratically convergent iterative algorithms of the form $z+$ $M\left(f(z), f^{\prime}(z)\right)$, which includes Newton's method as a special case. The motivation for this work is twofold. First, since the conditions for a point to be an approximate zero, given in [8,1, and 3], are basically the same (namely, $\gamma_{f}\left(z_{0}\right)\left|f\left(z_{0}\right)\right| /\left|f^{\prime}\left(z_{0}\right)\right|<\alpha_{0}$ for a certain constant $\alpha_{0}$ which varies among [8, 1 , $3]$ ), regardless of what iterative algorithm is under investigation (Newton's, Euler's, or Halley's), it is natural to ask to what extent this condition is universal, or in what form the condition would appear in a general setting. If the condition is indeed universal for a certain class of iterative algorithms, how does the constant $\alpha_{0}$ in the condition vary from one algorithm to another? In this paper, a similar condition for a point to be an approximate zero is established for the class of quadratically convergent algorithms. Moreover, it is shown how the bound $\alpha_{0}$ in the condition varies from one algorithm to another and what characteristic of the algorithm affects the bound. This puts the conditions found in $[8,1$, and 3] for Newton's method in a more general perspective. Second, other iterative algorithms of this form may be used to improve the convergence behavior of Newton's method in the global sense. According to the Fatou-Julia theory, each attracting cycle or fixed point attracts at least one critical point under the iteration of a rational map $z+M\left(f(z), f^{\prime}(z)\right)$; the nonconvergent behavior is essentially due to the existence of attracting cycles other than the fixed points (i.e., the zeros). So it may be possible to perturb Newton's method appropriately so as to remove some of the attracting cycles. In this way, in the region where Newton's method fails to converge to zeros, one may alter it to some other methods to achieve convergence. For example, Newton's method when applied to the polynomial $f(z)=\frac{1}{2} z^{3}-z+1$ has the attracting cycle $\{0,1\}$ on the real line. When using $M\left(f, f^{\prime}\right)=-f / f^{\prime}+f^{3} /\left(f^{\prime}\left(1+2 f^{2}\right)\right)$, the attracting cycle $\{0,1\}$ no longer exists. In fact, the whole real line is free of any attracting cycles. Therefore, to find a condition for a point to be an approximate zero for other quadratically convergent algorithms is important in its own right.

\section{NOTATIONS AND STATEMENTS OF THE RESULTS}

For a function $M: \mathbf{C} \times(\mathbf{C} \backslash\{0\}) \rightarrow \mathbf{C}$, consider iterative algorithms defined by

$$
I_{f}(z)=z+M\left(f(z), f^{\prime}(z)\right)
$$


for finding zeros of $f$, where $f$ can be any analytic function in the complex plane C. (We will work in this setting; but everything should readily carry over to the case of the real line or more general setting of Banach spaces with appropriate definitions and interpretations.) The assumptions we make on $M$ are the following:

(a) $M: \mathbf{C} \times(\mathbf{C} \backslash\{0\}) \rightarrow \mathbf{C}$ is analytic;

(b) $M(0, \cdot) \equiv 0$;

(c) $M^{(1,0)}(0, v)=-\frac{1}{v}$, where $M^{(1,0)}(u, v)=\frac{\partial M(u, v)}{\partial u}$.

Assumption (a) is reasonable; assumptions (b) and (c) are equivalent to the iteration $I_{f}$ being locally quadratically convergent at simple zeros of $f$. Newton's method is $M(u, v)=-u / v$, which clearly satisfies assumptions (a), (b), and (c).

We adopt most of the terminologies and notations used in [8], except that the notion of an approximate zero is defined here in terms of the residual and in a somewhat stronger sense.

Definition. We call $z_{0}$ an approximate zero of $f$ with respect to the iteration $I_{f}$ defined by $M$ if there exist positive numbers $a_{0}, a_{1}, a_{2}, \ldots$ satisfying $a_{0}<1$ and $a_{k+1} \leq a_{k}^{2}$ for all $k \geq 0$ such that

$$
\left|f\left(z_{n+k}\right)\right| \leq a_{k}^{2^{n}-1}\left|f\left(z_{k}\right)\right|, \quad n \geq 0, k \geq 0,
$$

where $z_{n}=I_{f}^{n}\left(z_{0}\right)$ for $n \geq 0$.

Remark. One of the advantages of our definition of an approximate zero is that it is forward-invariant, namely, if $z_{0}$ is an approximate zero, then so is $z_{1}=I_{f}\left(z_{0}\right)$. The original definition in [8] lacks this property. Another advantage is that it captures the full strength of quadratic convergence. It is easy to see that if $z_{0}$ is an approximate zero under our definition, then

$$
\left|f\left(z_{n}\right)\right| \leq a_{0}^{2^{n}-1}\left|f\left(z_{0}\right)\right|, \quad n \geq 0,
$$

which is the residual version of the original definition in [8] when $a_{0}=1 / 2$. It should be pointed out that the condition for a point to be an approximate zero found in [8] is still valid under our definition.

Let

$$
\begin{gathered}
\gamma_{f}(z)=\sup _{k>1}\left|\frac{f^{(k)}(z)}{k ! f^{\prime}(z)}\right|^{\frac{1}{k-1}}, \\
\tau_{M}(u, v)=\sup _{n_{1}+n_{2} \geq 1}\left|\frac{M^{\left(n_{1}, n_{2}\right)}(u, v)}{n_{1} ! n_{2} !}\right|^{\frac{1}{n_{1}+n_{2}}},
\end{gathered}
$$

where $M^{\left(n_{1}, n_{2}\right)}(u, v)=\partial^{n_{1}+n_{2}} M(u, v) / \partial u^{n_{1}} \partial v^{n_{2}}$.

Theorem 1. Let $M$ satisfy the assumptions (a), (b), and (c). Assume that

$$
K_{M} \stackrel{\text { def }}{=} \sup _{|v| \neq 0}|v| \tau_{M}(0, v)<\infty
$$




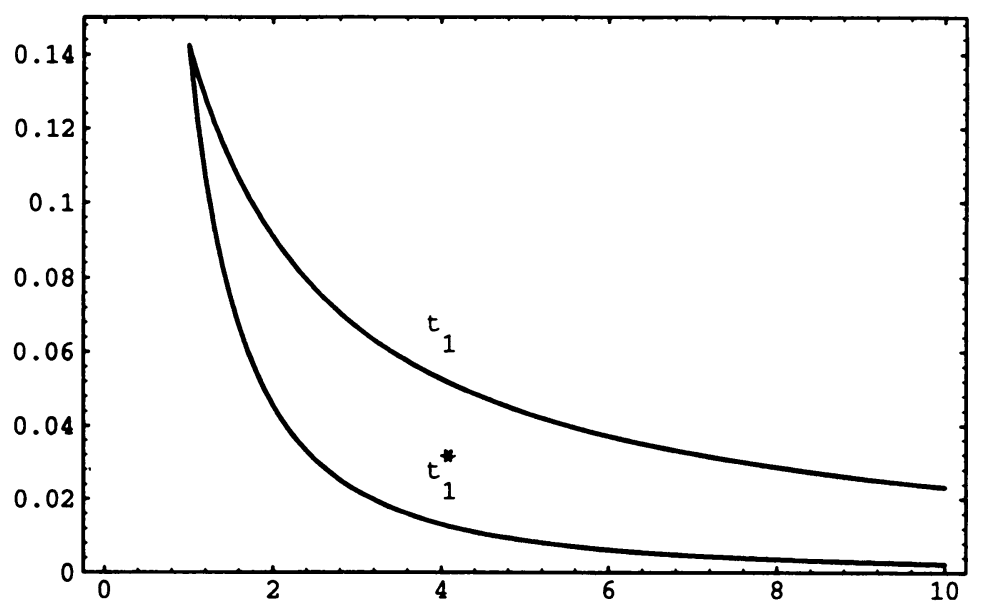

FIGURE 2.1

then there exists a number $t_{1}\left(K_{M}\right)$ depending on $K_{M}$ (in a way to be specified in the next section and pictured in Figure 2.1) such that if

$$
\left(\gamma_{f}\left(z_{0}\right)+1\right)\left|f\left(z_{0}\right)\right| \tau_{M}\left(0, f^{\prime}\left(z_{0}\right)\right)<t_{1}\left(K_{M}\right),
$$

then $z_{0}$ is an approximate zero of $f$ with respect to $M$, namely, there exist positive numbers $a_{0}, a_{1}, a_{2}, \ldots$ satisfying $a_{0}<1$ and $a_{k+1} \leq a_{k}^{2}$ for all $k \geq 0$ such that $\left|f\left(z_{n+k}\right)\right| \leq a_{k}^{2^{n}-1}\left|f\left(z_{k}\right)\right|$ for all $n \geq 0$ and $k \geq 0$, where $z_{n}=I_{f}^{n}\left(z_{0}\right)$.

Let $t_{1}^{*}\left(K_{M}\right)=t_{1}\left(K_{M}\right) / K_{M}$. The following is an immediate corollary to Theorem 1 .

Corollary 1. If

$$
\left(\gamma_{f}\left(z_{0}\right)+1\right)\left|\frac{f\left(z_{0}\right)}{f^{\prime}\left(z_{0}\right)}\right|<t_{1}^{*}\left(K_{M}\right),
$$

then $z_{0}$ is an approximate zero of $f$ with respect to $M$.

For Newton's method, it is easy to compute that $\tau_{M}(0, v)=1 /|v|$. So $K_{M}=1$, consequently $t_{1}^{*}(1)=t_{1}(1) \approx 0.142301$. So we have the following.

Corollary 2. For Newton's method, if

$$
\left(\gamma_{f}\left(z_{0}\right)+1\right)\left|\frac{f\left(z_{0}\right)}{f^{\prime}\left(z_{0}\right)}\right|<t_{1}(1) \approx 0.142301,
$$

then $z_{0}$ is an approximate zero of $f$ with respect to the Newton iteration.

Note that the general condition in Theorem 1 essentially specializes in this case to the condition obtained in $[8,1$, and 3] for Newton's method.

The functions $t_{1}\left(K_{M}\right)$ and $t_{1}^{*}\left(K_{M}\right)$ are decreasing in $K_{M}$, as pictured in Figure 2.1. This reveals how the bound in the condition for approximate zeros depends on the algorithm $M$; it depends on $M$ through the number $K_{M}$ in a decreasing manner.

As another example, consider the iteration defined by $M(u, v)=-u / v+$ $u^{2} / v^{2}$. It can be checked that $\tau_{M}(0, v)=\sqrt[5]{4} /|v|$. So $K_{M}=\sqrt[5]{4} \approx 1.3195$ and $t_{1}(\sqrt[5]{4}) \approx 0.120574$. Hence, we have the following corollary. 


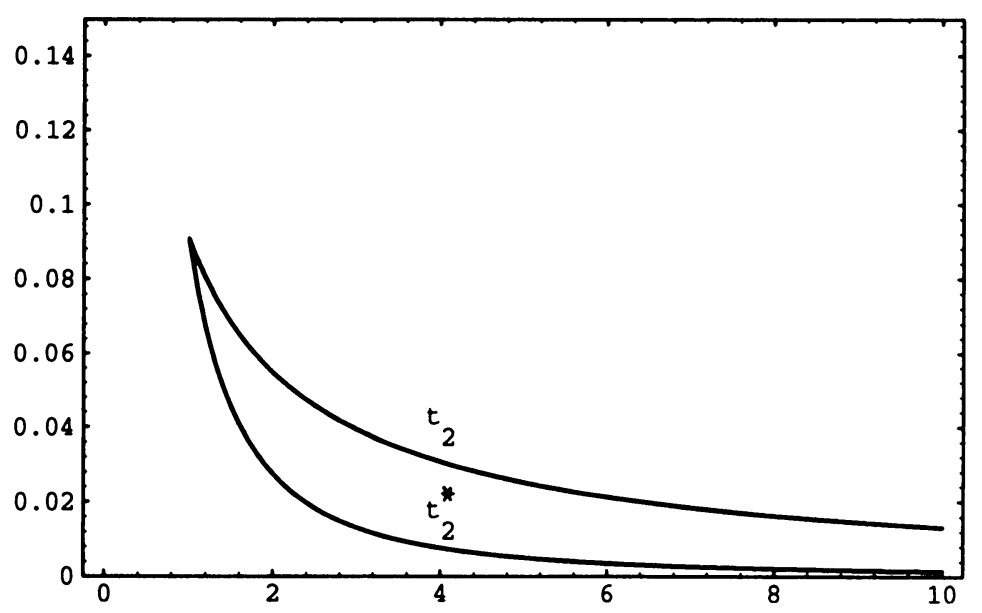

FIGURE 2.2

\section{Corollary 3. If}

$$
\left(\gamma_{f}\left(z_{0}\right)+1\right)\left|\frac{f\left(z_{0}\right)}{f^{\prime}\left(z_{0}\right)}\right|<t_{1}^{*}(\sqrt[5]{4}) \approx 0.091378,
$$

then $z_{0}$ is an approximate zero of $f$ with respect to the iteration defined by $M(u, v)=-u / v+u^{2} / v^{2}$.

Even though satisfied by Newton's method, the assumption that $K_{M}<\infty$ in Theorem 1 seems overly strong. Fortunately, it is by no means necessary. The following is an extension of Theorem 1 without that assumption.

Theorem 2. Let $M$ satisfy the assumptions (a), (b), and (c). There exists a function $t_{2}(r)$ (to be specified in $\S 4$ and pictured in Figure 2.2) such that if

$$
\left(\gamma_{f}\left(z_{0}\right)+1\right)\left|f\left(z_{0}\right)\right| \tau_{M}\left(0, f^{\prime}\left(z_{0}\right)\right)<t_{2}\left(\left|f^{\prime}\left(z_{0}\right)\right| \tau_{M}\left(0, f^{\prime}\left(z_{0}\right)\right)\right),
$$

then $z_{0}$ is an approximate zero of $f$ with respect to $M$, namely, there exist positive numbers $a_{0}, a_{1}, a_{2}, \ldots$ satisfying $a_{0}<1$ and $a_{k+1} \leq a_{k}^{2}$ for all $k \geq 0$ such that $\left|f\left(z_{n+k}\right)\right| \leq a_{k}^{2^{n}-1}\left|f\left(z_{k}\right)\right|$ for all $n \geq 0$ and $k \geq 0$, where $z_{n}=I_{f}^{n}\left(z_{0}\right)$.

Let $t_{2}^{*}(r)=t_{2}(r) / r$; then the condition in Theorem 2 can be rephrased as

$$
\left(\gamma_{f}\left(z_{0}\right)+1\right)\left|\frac{f\left(z_{0}\right)}{f^{\prime}\left(z_{0}\right)}\right|<t_{2}^{*}\left(\left|f^{\prime}\left(z_{0}\right)\right| \tau_{M}\left(0, f^{\prime}\left(z_{0}\right)\right)\right) .
$$

Again notice how the information about the algorithm $M$ enters the bound. The bound depends on the quantity $\left|f^{\prime}\left(z_{0}\right)\right| \tau_{M}\left(0, f^{\prime}\left(z_{0}\right)\right)$ in a decreasing manner, as pictured in Figure 2.2.

For Newton's method, $\left|f^{\prime}\left(z_{0}\right)\right| \tau_{M}\left(0, f^{\prime}\left(z_{0}\right)\right)=1$ for all $z_{0}$. So the bound given by Theorem 2 is $t_{2}^{*}(1)=t_{2}(1) \approx 0.090571$.

As an illustration for this case, consider the iteration defined by $M(u, v)=$ $-u / v+u^{2}$. It can be checked that

$$
\tau_{M}(0, v)=\max \{1,1 /|v|\} .
$$

Suppose that we want to find an approximate zero of $f(z)=z^{4}-z-1 / 100$. It can be calculated that $\gamma_{f}(1)=2$ and $\left|f^{\prime}(1)\right| \tau_{M}\left(0, f^{\prime}(1)\right)=3$. So the bound is 
$t_{2}^{*}(3) \approx 0.0131$. Note that $\left(\gamma_{f}(1)+1\right)|f(1)| /\left|f^{\prime}(1)\right|=0.01<0.0131$, so Theorem 2 guarantees that $z_{0}=1$ is an approximate zero of $f$ with respect to the $M$ specified above. Incidentally, note that the exact zero is about 1.00331135 . As another illustration, consider the function $g(z)=z e^{z}+z-1 / 30$. It can be verified that $\gamma_{g}(0)=1 / 2$ and $\left|g^{\prime}(0)\right| \tau_{M}\left(0, g^{\prime}(0)\right)=2$. So the bound is $t_{2}^{*}(2) \approx 0.0273788$. Since $\left(\gamma_{g}(0)+1\right)|g(0)| /\left|g^{\prime}(0)\right|=0.025<0.0273788$, by Theorem $2, z_{0}=0$ is an approximate zero of $g$ with respect to the given $M$. Again note that the exact zero of $g$ is about 0.0165289 .

\section{Proof of Theorem 1}

Let $z^{+}$denote $I_{f}(z)$ and $M_{z}$ denote $M\left(f(z), f^{\prime}(z)\right)$; then $z^{+}=z+M_{z}$.

Lemma 3.1. If $|u| \tau_{M}(0, v)<1$, then

(a) $M(u, v)=-\frac{u}{v}+\frac{M^{(2,0)}(0, v)}{2 !} u^{2}+\cdots+\frac{M^{(k, 0)}(0, v)}{k !} u^{k}+\cdots$;

(b) $|M(u, v)| \leq \frac{|u| \tau_{M}(0, \dot{v})}{1-|u| \tau_{M}(0, v)}$.

Proof. (a) This is Taylor expansion of $M(u, v)$ in $u$ at $u=0$. The radius of convergence is at least $1 / \tau_{M}(0, v)$ since $\left|M^{(k, 0)}(0, v)\right| / k ! \leq \tau_{M}(0, v)^{k}, k>$ 0 .

(b) is an immediate consequence of (a).

Lemma 3.2. If $|f(z)| \tau_{M}\left(0, f^{\prime}(z)\right)<1$, then

(a) $M\left(f(z), f^{\prime}(z)\right)=-\frac{f(z)}{f^{\prime}(z)}+\sum_{k=2}^{\infty} \frac{M^{(k, 0)}\left(0, f^{\prime}(z)\right)}{k !}(f(z))^{k}$;

(b) $\left|M\left(f(z), f^{\prime}(z)\right)\right| \leq \frac{|f(z)| \tau_{M}\left(0, f^{\prime}(z)\right)}{1-|f(z)| \tau_{M}\left(0, f^{\prime}(z)\right)}$.

Proof. This follows from Lemma 3.1 with $u=f(z), v=f^{\prime}(z)$.

Lemma 3.3. If $\left(\gamma_{f}(z)+1\right)|f(z)| \tau_{M}\left(0, f^{\prime}(z)\right)<1$, then

$$
\left|\frac{f\left(z^{+}\right)}{f(z)}\right| \leq \frac{\left(\gamma_{f}(z)+1\right)|f(z)|\left|f^{\prime}(z)\right| \tau_{M}\left(0, f^{\prime}(z)\right)^{2}}{1-\left(\gamma_{f}(z)+1\right)|f(z)| \tau_{M}\left(0, f^{\prime}(z)\right)} .
$$

Proof. By Lemma 3.2(b), $\left(\gamma_{f}(z)+1\right)|f(z)| \tau_{M}\left(0, f^{\prime}(z)\right)<1$ implies that $\left|M_{z}\right| \gamma_{f}(z)<1$. So the Taylor series

$$
f\left(z^{+}\right)=f\left(z+M_{z}\right)=\sum_{k=0}^{\infty} \frac{f^{(k)}(z)}{k !} M_{z}^{k}
$$

is convergent, since $\left|f^{(k)}(z)\right| / k ! \leq\left|f^{\prime}(z)\right|\left(\gamma_{f}(z)\right)^{k-1}$. Then

$$
\frac{f\left(z^{+}\right)}{f(z)}=\left(1+\frac{f^{\prime}(z)}{f(z)} M_{z}\right)+\frac{f^{\prime}(z)}{f(z)} \sum_{k=2}^{\infty} \frac{f^{(k)}(z)}{k ! f^{\prime}(z)} M_{z}^{k} .
$$

Now by Lemma 3.2(a), 


$$
\begin{aligned}
1+\frac{f^{\prime}(z)}{f(z)} M_{z} & =1+\frac{f^{\prime}(z)}{f(z)}\left[-\frac{f(z)}{f^{\prime}(z)}+\sum_{k=2}^{\infty} \frac{M^{(k, 0)}\left(0, f^{\prime}(z)\right)}{k !} f(z)^{k}\right] \\
& =\frac{f^{\prime}(z)}{f(z)} \sum_{k=2}^{\infty} \frac{M^{(k, 0)}\left(0, f^{\prime}(z)\right)}{k !} f(z)^{k} .
\end{aligned}
$$

So

$$
\begin{aligned}
\left|1+\frac{f^{\prime}(z)}{f(z)} M_{z}\right| & \leq\left|\frac{f^{\prime}(z)}{f(z)}\right| \sum_{k=2}^{\infty}\left|\frac{M^{(k, 0)}\left(0, f^{\prime}(z)\right)}{k !}\right||f(z)|^{k} \\
& \leq\left|\frac{f^{\prime}(z)}{f(z)}\right| \sum_{k=2}^{\infty} \tau_{M}\left(0, f^{\prime}(z)\right)^{k}|f(z)|^{k} \\
& =\left|\frac{f^{\prime}(z)}{f(z)}\right| \frac{|f(z)|^{2} \tau_{M}\left(0, f^{\prime}(z)\right)^{2}}{1-|f(z)| \tau_{M}\left(0, f^{\prime}(z)\right)} .
\end{aligned}
$$

By Lemma 3.2(b),

$$
\begin{aligned}
& \left|\frac{f^{\prime}(z)}{f(z)} \sum_{k=2}^{\infty} \frac{f^{(k)}(z)}{k ! f^{\prime}(z)} M_{z}^{k}\right| \leq\left|\frac{f^{\prime}(z)}{f(z)}\right| \sum_{k=2}^{\infty} \gamma_{f}(z)^{k-1}\left|M_{z}\right|^{k} \\
& =\left|\frac{f^{\prime}(z)}{f(z)}\right| \frac{\gamma_{f}(z)\left|M_{z}\right|^{2}}{1-\gamma_{f}(z)\left|M_{z}\right|} \leq\left|\frac{f^{\prime}(z)}{f(z)}\right| \frac{\gamma_{f}(z)\left[\frac{|f(z)| \tau_{M}\left(0, f^{\prime}(z)\right)}{1-|f(z)| \tau_{M}\left(0, f^{\prime}(z)\right)}\right]^{2}}{1-\gamma_{f}(z)\left[\frac{|f(z)| \tau_{M}\left(0, f^{\prime}(z)\right)}{1-|f(z)| \tau_{M}\left(0, f^{\prime}(z)\right)}\right]} \\
& =\left|\frac{f^{\prime}(z)}{f(z)}\right| \frac{\gamma_{f}(z)|f(z)|^{2} \tau_{M}\left(0, f^{\prime}(z)\right)^{2}}{1-\left(\gamma_{f}(z)+1\right)|f(z)| \tau_{M}\left(0, f^{\prime}(z)\right)} \frac{1}{1-|f(z)| \tau_{M}\left(0, f^{\prime}(z)\right)} .
\end{aligned}
$$

So (by abbreviating $f(z), f^{\prime}(z), \gamma_{f}(z), \tau_{M}\left(0, f^{\prime}(z)\right)$ as $f, f^{\prime}, \gamma_{f}, \tau_{M}$, respectively)

$$
\begin{aligned}
\left|\frac{f\left(z^{+}\right)}{f(z)}\right| & \leq\left|\frac{f^{\prime}}{f}\right| \frac{|f|^{2} \tau_{M}^{2}}{1-|f| \tau_{M}}+\left|\frac{f^{\prime}}{f}\right| \frac{\gamma_{f}|f|^{2} \tau_{M}^{2}}{1-\left(\gamma_{f}+1\right)|f| \tau_{M}} \frac{1}{1-|f| \tau_{M}} \\
& =\left|\frac{f^{\prime}}{f}\right| \frac{|f|^{2} \tau_{M}^{2}}{1-|f| \tau_{M}}\left(1+\frac{\gamma_{f}}{1-\left(\gamma_{f}+1\right)|f| \tau_{M}}\right) \\
& =\frac{\left(\gamma_{f}+1\right)|f|\left|f^{\prime}\right| \tau_{M}^{2}}{1-\left(\gamma_{f}+1\right)|f| \tau_{M}} .
\end{aligned}
$$

Lemma 3.4. (a) If $\left|M_{z}\right| \gamma_{f}(z)<1$, then

$$
\left|f^{\prime}\left(z^{+}\right)-f^{\prime}(z)\right| \leq\left|f^{\prime}(z)\right|\left[\frac{1}{\left(1-\gamma_{f}(z)\left|M_{z}\right|\right)^{2}}-1\right] \text {. }
$$

(b) If $\left(\gamma_{f}(z)+1\right)|f(z)| \tau_{M}\left(0, f^{\prime}(z)\right)<1$, then

$$
\left|f^{\prime}\left(z^{+}\right)\right| \leq \frac{\left|f^{\prime}(z)\right|\left[1-|f(z)| \tau_{M}\left(0, f^{\prime}(z)\right)\right]^{2}}{\left[1-\left(\gamma_{f}(z)+1\right)|f(z)| \tau_{M}\left(0, f^{\prime}(z)\right)\right]^{2}} .
$$


Proof. (a) Since $\left|M_{z}\right| \gamma_{f}(z)<1$, the Taylor series

$$
f^{\prime}\left(z^{+}\right)=f^{\prime}\left(z+M_{z}\right)=f^{\prime}(z)+\sum_{k=1}^{\infty} \frac{f^{(k+1)}(z)}{k !} M_{z}^{k}
$$

is convergent. Then

$$
\begin{aligned}
& \left|f^{\prime}\left(z^{+}\right)-f^{\prime}(z)\right|=\left|\sum_{k=1}^{\infty} \frac{f^{(k+1)}(z)}{k !} M_{z}^{k}\right| \leq\left|f^{\prime}(z)\right| \sum_{k=1}^{\infty}\left|\frac{f^{(k+1)}(z)}{k ! f^{\prime}(z)}\right|\left|M_{z}\right|^{k} \\
& \quad \leq\left|f^{\prime}(z)\right| \sum_{k=1}^{\infty}(k+1) \gamma_{f}(z)^{k}\left|M_{z}\right|^{k}=\left|f^{\prime}(z)\right|\left[\frac{1}{\left(1-\gamma_{f}(z)\left|M_{z}\right|\right)^{2}}-1\right] .
\end{aligned}
$$

(b) Again note that $\left(\gamma_{f}(z)+1\right)|f(z)| \tau_{M}\left(0, f^{\prime}(z)\right)<1$ implies that $\left|M_{z}\right| \gamma_{f}(z)$ $<1$ by Lemma 3.2(b). It follows from part (a) and Lemma 3.2(b) that

$$
\begin{aligned}
\left|f^{\prime}\left(z^{+}\right)\right| & \leq\left|f^{\prime}\left(z^{+}\right)-f^{\prime}(z)\right|+\left|f^{\prime}(z)\right| \leq \frac{\left|f^{\prime}(z)\right|}{\left(1-\gamma_{f}(z)\left|M_{z}\right|\right)^{2}} \\
& \leq \frac{\left|f^{\prime}(z)\right|\left[1-|f(z)| \tau_{M}\left(0, f^{\prime}(z)\right)\right]^{2}}{\left[1-\left(\gamma_{f}(z)+1\right)|f(z)| \tau_{M}\left(0, f^{\prime}(z)\right)\right]^{2}} .
\end{aligned}
$$

Lemma 3.5. If $|h| \tau_{M}(0, v)<1$, then

$$
\tau_{M}(0, v+h) \leq \frac{\tau_{M}(0, v)}{\left(1-|h| \tau_{M}(0, v)\right)^{2}} .
$$

Proof. For $n_{1} \geq 0, n_{2} \geq 0, n_{1}+n_{2} \geq 1$, expanding $M^{\left(n_{1}, n_{2}\right)}(0, \cdot)$ at $v$ into Taylor series, we have

$$
M^{\left(n_{1}, n_{2}\right)}(0, v+h)=\sum_{k=0}^{\infty} \frac{M^{\left(n_{1}, n_{2}+k\right)}(0, v)}{k !} h^{k}, \quad|h|<\frac{1}{\tau_{M}(0, v)} .
$$

Then

$$
\begin{aligned}
& \frac{\left|M^{\left(n_{1}, n_{2}\right)}(0, v+h)\right|}{n_{1} ! n_{2} !} \leq \sum_{k=0}^{\infty} \frac{\left(n_{2}+k\right) !}{n_{2} ! k !} \frac{\left|M^{\left(n_{1}, n_{2}+k\right)}(0, v)\right|}{n_{1} !\left(n_{2}+k\right) !}|h|^{k} \\
& \quad \leq \sum_{k=0}^{\infty} \frac{\left(n_{2}+k\right) !}{n_{2} ! k !} \tau_{M}(0, v)^{n_{1}+n_{2}+k}|h|^{k} \\
& \quad=\frac{\tau_{M}(0, v)^{n_{1}+n_{2}}}{n_{2} !} \sum_{k=0}^{\infty}\left(n_{2}+k\right) \cdots(k+1)\left(|h| \tau_{M}(0, v)\right)^{k} \\
& \quad=\frac{\tau_{M}(0, v)^{n_{1}+n_{2}}}{n_{2} !} \frac{n_{2} !}{\left(1-|h| \tau_{M}(0, v)\right)^{n_{2}+1}}=\frac{\tau_{M}(0, v)^{n_{1}+n_{2}}}{\left(1-|h| \tau_{M}(0, v)\right)^{n_{2}+1}} .
\end{aligned}
$$

So

$$
\begin{aligned}
\tau_{M}(0, v+h) & =\sup _{n_{1}+n_{2}>0}\left|\frac{M^{\left(n_{1}, n_{2}\right)}(0, v+h)}{n_{1} ! n_{2} !}\right|^{\frac{1}{n_{1}+n_{2}}} \\
& \leq \sup _{n_{1}+n_{2}>0} \frac{\tau_{M}(0, v)}{\left(1-|h| \tau_{M}(0, v)\right)^{\frac{n_{2}+1}{n_{1}+n_{2}}}}=\frac{\tau_{M}(0, v)}{\left(1-|h| \tau_{M}(0, v)\right)^{2}}
\end{aligned}
$$


Lemma 3.6. If

$$
\frac{\left(\gamma_{f}(z)+1\right)|f(z)| \tau_{M}\left(0, f^{\prime}(z)\right)}{1-\left(\gamma_{f}(z)+1\right)|f(z)| \tau_{M}\left(0, f^{\prime}(z)\right)}<1-\sqrt{\frac{K_{M}}{1+K_{M}}}
$$

(i.e., $\left.\left(\gamma_{f}(z)+1\right)|f(z)| \tau_{M}\left(0, f^{\prime}(z)\right)<\left(1-\sqrt{\frac{K_{M}}{1+K_{M}}}\right) /\left(2-\sqrt{\frac{K_{M}}{1+K_{M}}}\right)\right)$, then

$$
\tau_{M}\left(0, f^{\prime}\left(z^{+}\right)\right) \leq \frac{\tau_{M}\left(0, f^{\prime}(z)\right)\left[1-\left(\gamma_{f}(z)+1\right)|f(z)| \tau_{M}\left(0, f^{\prime}(z)\right)\right]^{4}}{\left\{\left(1+K_{M}\right)\left[1-\left(\gamma_{f}(z)+1\right)|f(z)| \tau_{M}\left(0, f^{\prime}(z)\right)\right]^{2}-K_{M}\right\}^{2}} .
$$

Proof. Let $\gamma_{f}(z), f(z), \tau_{M}\left(0, f^{\prime}(z)\right)$ be abbreviated as $\gamma_{f}, f, \tau_{M}$, respectively. Since

$$
|f| \tau_{M} \leq\left(\gamma_{f}+1\right)|f| \tau_{M}<\frac{1-\sqrt{\frac{K_{M}}{1+K_{M}}}}{2-\sqrt{\frac{K_{M}}{1+K_{M}}}}<1
$$

by Lemma 3.2(b),

$$
\gamma_{f}\left|M_{z}\right| \leq \frac{\gamma_{f}|f| \tau_{M}}{1-|f| \tau_{M}} \leq \frac{\left(\gamma_{f}+1\right)|f| \tau_{M}}{1-\left(\gamma_{f}+1\right)|f| \tau_{M}}<1-\sqrt{\frac{K_{M}}{1+K_{M}}}
$$

So $1 /\left(1-\gamma_{f}\left|M_{z}\right|\right)^{2}-1<1 / K_{M}$. If $f^{\prime}\left(z^{+}\right)=f^{\prime}(z)+\theta(z)$, then by Lemma 3.4(a),

$$
|\theta(z)| \tau_{M}\left(0, f^{\prime}(z)\right) \leq\left|f^{\prime}\right| \tau_{M}\left[\frac{1}{\left(1-\gamma_{f}\left|M_{z}\right|\right)^{2}}-1\right]<K_{M} \frac{1}{K_{M}}=1 .
$$

Now by Lemma 3.5, Lemma 3.4, and Lemma 3.2,

$$
\begin{aligned}
\tau_{M}(0 & \left., f^{\prime}\left(z^{+}\right)\right)=\tau_{M}\left(0, f^{\prime}(z)+\theta(z)\right) \leq \frac{\tau_{M}\left(0, f^{\prime}(z)\right)}{\left[1-|\theta(z)| \tau_{M}\left(0, f^{\prime}(z)\right)\right]^{2}} \\
& \leq \frac{\tau_{M}}{\left\{1-\left|f^{\prime}\right| \tau_{M}\left[\frac{1}{\left(1-\gamma_{f}\left|M_{z}\right|\right)^{2}}-1\right]\right\}^{2}} \\
& \leq \frac{\tau_{M}}{\left\{1-\left|f^{\prime}\right| \tau_{M}\left[\frac{1}{\left(1-\gamma_{f}|f| \tau_{M} /\left(1-|f| \tau_{M}\right)\right)^{2}}-1\right]\right\}^{2}} \\
& =\frac{\tau_{M}\left(1-\left(\gamma_{f}+1\right)|f| \tau_{M}\right)^{4}}{\left\{\left(1-\left(\gamma_{f}+1\right)|f| \tau_{M}\right)^{2}-\left|f^{\prime}\right| \tau_{M}\left[\left(1-|f| \tau_{M}\right)^{2}-\left(1-\left(\gamma_{f}+1\right)|f| \tau_{M}\right)^{2}\right]\right\}^{2}} \\
& \leq \frac{\tau_{M}\left(1-\left(\gamma_{f}+1\right)|f| \tau_{M}\right)^{4}}{\left\{\left(1-\left(\gamma_{f}+1\right)|f| \tau_{M}\right)^{2}-K_{M}\left[1-\left(1-\left(\gamma_{f}+1\right)|f| \tau_{M}\right)^{2}\right]\right\}^{2}} \\
& =\frac{\tau_{M}\left(1-\left(\gamma_{f}+1\right)|f| \tau_{M}\right)^{4}}{\left[\left(1+K_{M}\right)\left(1-\left(\gamma_{f}+1\right)|f| \tau_{M}\right)^{2}-K_{M}\right]^{2}} . \square
\end{aligned}
$$


Lemma 3.7 ([8, Lemma 2 of $\S 3])$. For all $z, z_{1}$, if $\left|z_{1}-z\right| \gamma_{f}(z)<1-\sqrt{2} / 2$, then

$$
\gamma_{f}\left(z_{1}\right) \leq \frac{\gamma_{f}(z)}{\left[2\left(1-\left|z_{1}-z\right| \gamma_{f}(z)\right)^{2}-1\right]\left(1-\left|z_{1}-z\right| \gamma_{f}(z)\right)} .
$$

Corollary. If $\gamma_{f}(z)\left|M_{z}\right|<1-\sqrt{2} / 2$, then

$$
\gamma_{f}\left(z^{+}\right) \leq \frac{\gamma_{f}(z)}{\left[2\left(1-\gamma_{f}(z)\left|M_{z}\right|\right)^{2}-1\right]\left(1-\gamma_{f}(z)\left|M_{z}\right|\right)} .
$$

Proof. This follows from Lemma 3.7 with $z_{1}=z^{+}$.

Lemma 3.8. If

$$
\frac{\left(\gamma_{f}(z)+1\right)|f(z)| \tau_{M}\left(0, f^{\prime}(z)\right)}{1-\left(\gamma_{f}(z)+1\right)|f(z)| \tau_{M}\left(0, f^{\prime}(z)\right)}<1-\frac{\sqrt{2}}{2}
$$

(i.e., $\left.\left(\gamma_{f}+1\right)|f| \tau_{M}<\left(1-\frac{\sqrt{2}}{2}\right) /\left(2-\frac{\sqrt{2}}{2}\right)\right)$, then

(a) $\gamma_{f}\left(z^{+}\right) \leq \frac{\gamma_{f}}{\left[2\left(1-\left(\gamma_{f}+1\right)|f| \tau_{M}\right)^{2}-1\right]\left[1-\left(\gamma_{f}+1\right)|f| \tau_{M}\right]}$;

(b) $\gamma_{f}\left(z^{+}\right)+1 \leq \frac{\gamma_{f}+1}{\left[2\left(1-\left(\gamma_{f}+1\right)|f| \tau_{M}\right)^{2}-1\right]\left[1-\left(\gamma_{f}+1\right)|f| \tau_{M}\right]}$,

where $\gamma_{f}=\gamma_{f}(z), f=f(z)$, and $\tau_{M}=\tau_{M}\left(0, f^{\prime}(z)\right)$ as before.

Proof. (a) By Lemma 3.2(b),

$$
\gamma_{f}\left|M_{z}\right| \leq \frac{\gamma_{f}|f| \tau_{M}}{1-|f| \tau_{M}}<\frac{\left(\gamma_{f}+1\right)|f| \tau_{M}}{1-\left(\gamma_{f}+1\right)|f| \tau_{M}}<1-\frac{\sqrt{2}}{2} .
$$

By the Corollary to Lemma 3.7,

$$
\begin{aligned}
\gamma_{f}\left(z^{+}\right) & \leq \frac{\gamma_{f}(z)}{\left[2\left(1-\gamma_{f}\left|M_{z}\right|\right)^{2}-1\right]\left(1-\gamma_{f}\left|M_{z}\right|\right)} \\
& \leq \frac{\gamma_{f}(z)}{\left[2\left(1-\frac{\gamma_{f}|f| \tau_{M}}{1-|f| \tau_{M}}\right)^{2}-1\right]\left(1-\frac{\gamma_{f}|f| \tau_{M}}{1-|f| \tau_{M}}\right)} \\
& =\frac{\gamma_{f}(z)\left(1-|f| \tau_{M}\right)^{3}}{\left[2\left(1-\left(\gamma_{f}+1\right)|f| \tau_{M}\right)^{2}-\left(1-|f| \tau_{M}\right)^{2}\right]\left[1-\left(\gamma_{f}+1\right)|f| \tau_{M}\right]} \\
& \leq \frac{\gamma_{f}(z)}{\left[2\left(1-\left(\gamma_{f}+1\right)|f| \tau_{M}\right)^{2}-1\right]\left[1-\left(\gamma_{f}+1\right)|f| \tau_{M}\right]} .
\end{aligned}
$$

(b) This follows from part (a) and the fact that $1 /\left[\left(2(1-x)^{2}-1\right)(1-x)\right] \geq$ $1, \forall x \in[0,1-\sqrt{2} / 2)$. 


$$
\begin{aligned}
& \phi_{1}(x)=\frac{1}{1-x}, \\
& \phi_{2}\left(x, K_{M}\right)=\frac{(1-x)^{4}}{\left[\left(1+K_{M}\right)(1-x)^{2}-K_{M}\right]^{2}}, \\
& \phi_{3}(x)=\frac{1}{\left[2(1-x)^{2}-1\right](1-x)} \\
& \phi\left(x, K_{M}\right)=K_{M} \phi_{1}(x) \phi_{2}\left(x, K_{M}\right) \phi_{3}(x) \\
& \quad=\frac{K_{M}(1-x)^{2}}{\left[\left(1+K_{M}\right)(1-x)^{2}-K_{M}\right]^{2}\left[2(1-x)^{2}-1\right]}, \\
& \psi_{1}\left(x, K_{M}\right)=x \phi\left(x, K_{M}\right)=\frac{K_{M} x(1-x)^{2}}{\left[\left(1+K_{M}\right)(1-x)^{2}-K_{M}\right]^{2}\left[2(1-x)^{2}-1\right]}
\end{aligned}
$$

It can be checked that $\phi\left(x, K_{M}\right)$ is increasing in $x$ on $\left[0,1-\sqrt{\frac{K_{M}}{1+K_{M}}}\right)$ (note that $1-\sqrt{\frac{K_{M}}{1+K_{M}}} \leq 1-\frac{\sqrt{2}}{2}$ since $K_{M} \geq 1$ ). So $\psi_{1}\left(\cdot, K_{M}\right)$ maps $[0,1-$ $\left.\sqrt{\frac{K_{M}}{1+K_{M}}}\right)$ monotonically onto $[0, \infty)$ for each $K_{M} \geq 1$. Let $t_{1}\left(K_{M}\right)$ denote the unique number in $\left[0,1-\sqrt{\frac{K_{M}}{1+K_{M}}}\right)$ such that $\psi_{1}\left(t_{1}\left(K_{M}\right), K_{M}\right)=1$.

Lemma 3.9. There holds

$$
0<t_{1}\left(K_{M}\right)<\frac{1-\sqrt{\frac{K_{M}}{1+K_{M}}}}{2-\sqrt{\frac{K_{M}}{1+K_{M}}}}, \quad K_{M} \geq 1 .
$$

The proof of this lemma is left to the appendix since it does not shed any light on later developments.

Let $T_{f}(z)=\left(\gamma_{f}(z)+1\right)|f(z)| \tau_{M}\left(0, f^{\prime}(z)\right)$.

Lemma 3.10. If $z_{0}$ satisfies $T_{f}\left(z_{0}\right)<t_{1}\left(K_{M}\right)$, then there exist positive numbers $a_{0}, a_{1}, a_{2}, \ldots$ satisfying $a_{0}<1$ and $a_{k+1} \leq a_{k}^{2}$ for all $k \geq 0$ such that $T_{f}\left(z_{n+k}\right) \leq a_{k}^{2^{n}-1} T_{f}\left(z_{k}\right)$ for all $n \geq 0$ and $k \geq 0$, where $z_{n}=I_{f}^{n}\left(z_{0}\right), n \geq 0$.

Proof. By Lemma 3.9 and Lemma 3.3, we have

$$
\left|f\left(z_{1}\right)\right| \leq \frac{\left|f^{\prime}\left(z_{0}\right)\right|}{\gamma_{f}\left(z_{0}\right)+1} \phi_{1}\left(T_{f}\left(z_{0}\right)\right)\left(T_{f}\left(z_{0}\right)\right)^{2} .
$$

By Lemma 3.9 and Lemma 3.6, we have

$$
\tau_{M}\left(0, f^{\prime}\left(z_{1}\right)\right) \leq \tau_{M}\left(0, f^{\prime}\left(z_{0}\right)\right) \phi_{2}\left(T_{f}\left(z_{0}\right), K_{M}\right) .
$$

By Lemma 3.9 and Lemma 3.8(b), we have

$$
\gamma_{f}\left(z_{1}\right)+1 \leq\left(\gamma_{f}\left(z_{0}\right)+1\right) \phi_{3}\left(T_{f}\left(z_{0}\right)\right) .
$$

Combining (3.1), (3.2), (3.3) and using the definitions of $K_{M}$ and $\phi$, we have

$$
T_{f}\left(z_{1}\right) \leq \phi\left(T_{f}\left(z_{0}\right), K_{M}\right)\left(T_{f}\left(z_{0}\right)\right)^{2} .
$$

Let $a_{0}=\psi_{1}\left(T_{f}\left(z_{0}\right), K_{M}\right)$; then $T_{f}\left(z_{1}\right) \leq a_{0} T_{f}\left(z_{0}\right)$. Since $T_{f}\left(z_{0}\right)<t_{1}\left(K_{M}\right)$, we have $a_{0}<1$. Inductively, we have 


$$
\begin{aligned}
& T_{f}\left(z_{n+1}\right) \leq a_{0} T_{f}\left(z_{n}\right), \quad n \geq 0, \\
& T_{f}\left(z_{n+1}\right) \leq \phi\left(T_{f}\left(z_{n}\right), K_{M}\right)\left(T_{f}\left(z_{n}\right)\right)^{2}, \quad n \geq 0 .
\end{aligned}
$$

Hence, it follows that

$$
T_{f}\left(z_{n}\right) \leq\left[T_{f}\left(z_{0}\right) \phi\left(T_{f}\left(z_{0}\right), K_{M}\right)\right]^{2^{n}-1} T_{f}\left(z_{0}\right)=a_{0}^{2^{n}-1} T_{f}\left(z_{0}\right), \quad n \geq 0 .
$$

To see this, we use induction on $n$ and note that $\phi\left(\cdot, K_{M}\right)$ is increasing and $T_{f}\left(z_{n}\right)<T_{f}\left(z_{0}\right)$. So assuming (3.5) holds for $n$, we see from the following that it also holds for $n+1$ :

$$
\begin{aligned}
T_{f}\left(z_{n+1}\right) & \leq \phi\left(T_{f}\left(z_{n}\right), K_{M}\right)\left(T_{f}\left(z_{n}\right)\right)^{2} \\
& \leq \phi\left(T_{f}\left(z_{0}\right), K_{M}\right)\left[T_{f}\left(z_{0}\right) \phi\left(T_{f}\left(z_{0}\right), K_{M}\right)\right]^{2^{n+1}-2}\left(T_{f}\left(z_{0}\right)\right)^{2} \\
& =\left[T_{f}\left(z_{0}\right) \phi\left(T_{f}\left(z_{0}\right), K_{M}\right)\right]^{2^{n+1}-1} T_{f}\left(z_{0}\right)=a_{0}^{2^{n+1}-1} T_{f}\left(z_{0}\right) .
\end{aligned}
$$

Now for each $k \geq 1$, let $a_{k}=\psi_{1}\left(T_{f}\left(z_{k}\right), K_{M}\right)$; then we have $a_{k+1} \leq a_{k}^{2}$ for all $k \geq 0$. Indeed, since $T_{f}\left(z_{k+1}\right)<T_{f}\left(z_{k}\right)$ and $\phi\left(\cdot, K_{M}\right)$ is increasing,

$$
\begin{aligned}
a_{k+1} & =\psi_{1}\left(T_{f}\left(z_{k+1}\right), K_{M}\right)=T_{f}\left(z_{k+1}\right) \phi\left(T_{f}\left(z_{k+1}\right), K_{M}\right) \\
& \leq \phi\left(T_{f}\left(z_{k}\right), K_{M}\right)\left(T_{f}\left(z_{k}\right)\right)^{2} \phi\left(T_{f}\left(z_{k}\right), K_{M}\right)=\left(\psi_{1}\left(T_{f}\left(z_{k}\right), K_{M}\right)\right)^{2}=a_{k}^{2} .
\end{aligned}
$$

Since $T_{f}\left(z_{k}\right)<t_{1}\left(K_{M}\right)$ for each $k \geq 0$, the argument above can be applied to $z_{k}$ in place of $z_{0}$ as the initial point to conclude that $T_{f}\left(z_{n+k}\right) \leq a_{k}^{2^{n}-1} T_{f}\left(z_{k}\right)$, $n \geq 0$.

Theorem 1. If $z_{0}$ satisfies $T_{f}\left(z_{0}\right)<t_{1}\left(K_{M}\right)$, then $z_{0}$ is an approximate zero of $f$ with respect to $M$, namely, there exist positive numbers $a_{0}, a_{1}, a_{2}, \ldots$ satisfying $a_{0}<1$ and $a_{k+1} \leq a_{k}^{2}$ for all $k \geq 0$ such that

$$
\left|f\left(z_{n+k}\right)\right| \leq a_{k}^{2^{n}-1}\left|f\left(z_{k}\right)\right|, \quad n \geq 0, k \geq 0 .
$$

Proof. Let $a_{0}, a_{1}, a_{2}, \ldots$ be as defined in Lemma 3.10; then from Lemma 3.10 we have $a_{k+1} \leq a_{k}^{2}$ for all $k \geq 0$ and

$$
T_{f}\left(z_{n+k}\right) \leq a_{k}^{2^{n}-1} T_{f}\left(z_{k}\right), \quad n \geq 0, k \geq 0 .
$$

By Lemma 3.3, we have

$$
\left|f\left(z_{n}\right)\right| \leq\left|f^{\prime}\left(z_{n-1}\right)\right| \tau_{M}\left(0, f^{\prime}\left(z_{n-1}\right)\right) \frac{T_{f}\left(z_{n-1}\right)}{1-T_{f}\left(z_{n-1}\right)}\left|f\left(z_{n-1}\right)\right|, \quad n \geq 1 .
$$

Using these facts, we prove the theorem by induction on $n$ for each $k \geq 0$. When $n=0$, the inequality to be proved clearly holds. Suppose we have $\left|f\left(z_{n+k}\right)\right| \leq a_{k}^{2^{n}-1}\left|f\left(z_{k}\right)\right|$; then by using (3.6), (3.7), and the definition of $K_{M}$, we see

$$
\begin{aligned}
\left|f\left(z_{n+k+1}\right)\right| & \leq\left|f^{\prime}\left(z_{n+k}\right)\right| \tau_{M}\left(0, f^{\prime}\left(z_{n+k}\right)\right) \frac{T_{f}\left(z_{n+k}\right)}{1-T_{f}\left(z_{n+k}\right)}\left|f\left(z_{n+k}\right)\right| \\
& \leq K_{M} \frac{a_{k}^{2^{n}-1} T_{f}\left(z_{k}\right)}{1-a_{k}^{2^{n}-1} T_{f}\left(z_{k}\right)} a_{k}^{2^{n}-1}\left|f\left(z_{k}\right)\right| \leq a_{k}^{2^{n+1}-2} \frac{K_{M} T_{f}\left(z_{k}\right)}{1-T_{f}\left(z_{k}\right)}\left|f\left(z_{k}\right)\right| \\
& \leq a_{k}^{2^{n+1}-1}\left|f\left(z_{k}\right)\right| .
\end{aligned}
$$


The last inequality follows from the fact that

$$
\frac{K_{M} T_{f}\left(z_{k}\right)}{1-T_{f}\left(z_{k}\right)} \leq T_{f}\left(z_{k}\right) \phi\left(T_{f}\left(z_{k}\right), K_{M}\right)=a_{k} .
$$

To see that, note that the function $(1-x) \phi\left(x, K_{M}\right)$ is increasing in $x$ on $\left[0,1-\sqrt{K_{M} /\left(1+K_{M}\right)}\right)$ for each $K_{M} \geq 1$. So $(1-x) \phi\left(x, K_{M}\right) \geq \phi\left(0, K_{M}\right)=$ $K_{M}$, or $K_{M} /(1-x) \leq \phi\left(x, K_{M}\right)$ on $\left[0,1-\sqrt{K_{M} /\left(1+K_{M}\right)}\right)$.

For Newton's method, $M(u, v)=-u / v, \tau_{M}(0, v)=1 /|v|$, and $K_{M}=1$. So $\psi_{1}(x, 1)=x(1-x)^{2} /\left[2(1-x)^{2}-1\right]^{3}$ and $t_{1}(1) \approx 0.142301$. The condition in Theorem 1 specializes in this case to the following:

$$
\left(\gamma_{f}\left(z_{0}\right)+1\right)\left|\frac{f\left(z_{0}\right)}{f^{\prime}\left(z_{0}\right)}\right|<t_{1}^{*}(1)=t_{1}(1) \approx 0.142301 \text {. }
$$

For the iteration defined by $M(u, v)=-u / v+u^{2} / v^{2}$, we have $\tau_{M}(0, v)=$ $\sqrt[5]{4} /|v|$ and $K_{M}=\sqrt[5]{4}$. So

$$
\psi_{1}(x, \sqrt[5]{4})=\frac{\sqrt[5]{4} x(1-x)^{2}}{\left[\left((1+\sqrt[5]{4})(1-x)^{2}-\sqrt[5]{4}\right)^{2}\left(2(1-x)^{2}-1\right)\right]}
$$

and $t_{1}(\sqrt[5]{4}) \approx 0.120574$. The constant bound in the condition in this case is $t_{1}^{*}(\sqrt[5]{4})=t_{1}(\sqrt[5]{4}) / \sqrt[5]{4} \approx 0.091378$.

From the proof of Lemma 3.10, we see that $a_{0}=\psi_{1}\left(T_{f}\left(z_{0}\right), K_{M}\right)$. So for Newton's method, in order to have $a_{0} \leq 1 / 2$ as in Smale's definition of an approximate zero, we need to have $\left(\gamma_{f}\left(z_{0}\right)+1\right)\left|f\left(z_{0}\right)\right| /\left|f^{\prime}\left(z_{0}\right)\right| \leq \alpha \approx 0.115354$, where $\alpha$ satisfies $\psi_{1}(\alpha, 1)=1 / 2$. Notice that the constant bound obtained from our more general result is a little smaller than that in Smale's condition (which has been improved upon by Rheinboldt [10] by using the Kantorovich theory). This is probably the price that one usually has to pay for being more general. When comparing these results, one should also bear in mind that the definitions of an approximate zero upon which the results are derived are technically not quite the same.

\section{Proof of Theorem 2}

Let $r_{M}(z)=\left|f^{\prime}(z)\right| \tau_{M}\left(0, f^{\prime}(z)\right), z^{+}=I_{f}(z)$, and

$$
T_{f}(z)=\left(\gamma_{f}(z)+1\right)|f(z)| \tau_{M}\left(0, f^{\prime}(z)\right)
$$

as in $\S 3$. Lemma 3.6 can be rephrased in the following form.

Lemma 4.1. If $T_{f}(z) /\left(1-T_{f}(z)\right)<1-\sqrt{r_{M}(z) /\left(1+r_{M}(z)\right)}$, then

$$
\tau_{M}\left(0, f^{\prime}\left(z^{+}\right)\right) \leq \frac{\tau_{M}\left(0, f^{\prime}(z)\right)\left(1-T_{f}(z)\right)^{4}}{\left[\left(1+r_{M}(z)\right)\left(1-T_{f}(z)\right)^{2}-r_{M}(z)\right]^{2}} .
$$

Proof. The proof is virtually the same as that of Lemma 3.6 with $K_{M}$ being replaced by $r_{M}(z)$.

Combining Lemma 3.4(b) and Lemma 4.1, we have the following estimate on $r_{M}\left(z^{+}\right)$. 
Lemma 4.2. If $T_{f}(z) /\left(1-T_{f}(z)\right)<1-\sqrt{r_{M}(z) /\left(1+r_{M}(z)\right)}$, then

$$
r_{M}\left(z^{+}\right) \leq \frac{r_{M}(z)\left(1-T_{f}(z)\right)^{2}}{\left[\left(1+r_{M}(z)\right)\left(1-T_{f}(z)\right)^{2}-r_{M}(z)\right]^{2}} .
$$

From the proof of Lemma 3.10, we have the following estimate on $T_{f}\left(z^{+}\right)$.

Lemma 4.3. If $T_{f}(z) /\left(1-T_{f}(z)\right)<1-\sqrt{r_{M}(z) /\left(1+r_{M}(z)\right)}$, then

$$
T_{f}\left(z^{+}\right) \leq \frac{r_{M}(z)\left(T_{f}(z)\right)^{2}\left(1-T_{f}(z)\right)^{2}}{\left[\left(1+r_{M}(z)\right)\left(1-T_{f}(z)\right)^{2}-r_{M}(z)\right]^{2}\left[2\left(1-T_{f}(z)\right)^{2}-1\right]} .
$$

The following four technical lemmas are also needed for the proof of Theorem 2. Since their proofs are either somewhat lengthy or have little to do with the development of the proof of the theorem itself, they are consigned to the appendix.

For $r \geq 1$ and $m \geq 1$, let $u(r, m)$ be the positive solution of the equation

$$
\frac{u}{(1+r) u^{2}-r}=\sqrt{m}
$$

then it is easy to see that

$$
u(r, m)=\frac{1+\sqrt{1+4 m r(1+r)}}{2 \sqrt{m}(1+r)} .
$$

Lemma 4.4. There exists a constant $c>1$ such that $u(r, m)>\sqrt{m r /(1+m r)}$ for all $r \geq 1$ and $1 \leq m \leq c$. In fact, $c=(3+\sqrt{5}) / 2 \approx 2.618$ will do.

For $r \geq 1,1 \leq m \leq c(c=(3+\sqrt{5}) / 2)$, and $0 \leq a \leq 1$, let

$$
g(r, m, a)=\frac{1-a+a u(r, m)}{(1+m r)(1-a+a u(r, m))^{2}-m r},
$$

where $u(r, m)$ is defined above. Notice that in the specified domain, the function $g$ is well defined, i.e., the denominator is nonzero (in fact, positive) by Lemma 4.4 .

\section{Lemma 4.5.}

(a) $g(r, m, a)$ is increasing in $r$ on $[1, \infty)$ if $1 \leq m \leq c, 0 \leq a \leq 1$;

(b) $\lim _{r \rightarrow \infty} g(r, m, a)=\frac{1}{1+a \sqrt{m}-a m}$ if $1 \leq m \leq c, 0 \leq a \leq 1$;

(c) $1 \leq g(r, m, a) \leq \frac{1}{1+a \sqrt{m}-a m}$ if $1 \leq m \leq c, 0 \leq a \leq 1$.

\section{Lemma 4.6.}

(a) $1+a \sqrt{m}-a m>0$ if $1 \leq m<\frac{1}{2}+\frac{1}{a}+\sqrt{\frac{1}{4}+\frac{1}{a}}$;

(b) $\frac{1}{1+a \sqrt{m}-a m} \leq \sqrt{m}$ if $1 \leq m \leq \frac{1}{a}, 0 \leq a \leq 1$.

Let

$$
m(x, r)=\frac{(1-x)^{2}}{\left[(1+r)(1-x)^{2}-r\right]^{2}},
$$




$$
\psi_{2}(x, r)=\psi_{1}(x, r)(m(x, r))^{2}=\frac{r x(1-x)^{6}}{\left[(1+r)(1-x)^{2}-r\right]^{6}\left[2(1-x)^{2}-1\right]} .
$$

Then $\psi_{2}(x, r)$ is increasing in $x$ on $[0,1-\sqrt{r /(1+r)})$ and maps $[0,1-$ $\sqrt{r /(1+r)})$ onto $[0, \infty)$. Let $t_{2}(r)$ denote the unique number in $[0,1-$ $\sqrt{r /(1+r)})$ such that $\psi_{2}\left(t_{2}(r), r\right)=1$.

\section{Lemma 4.7.}

(a) $t_{2}(r)<t_{1}(r)$ for all $r \geq 1$;

(b) $m\left(t_{2}(r), r\right) \leq c(c=(3+\sqrt{5}) / 2)$ for all $r \geq 1$.

Lemma 4.8. If $T_{f}\left(z_{0}\right)<t_{2}\left(r_{M}\left(z_{0}\right)\right)$, then there exist positive numbers $a_{0}, a_{1}, \ldots$ satisfying $a_{0}<1$ and $a_{k+1} \leq a_{k}^{2}$ for all $k \geq 0$ such that $T_{f}\left(z_{n+k}\right) \leq a_{k}^{2^{n}-1} T_{f}\left(z_{k}\right)$ for all $n \geq 0$ and $k \geq 0$, where $z_{n}=T_{f}^{n}\left(z_{0}\right)$.

Proof. Let $m_{k}=m\left(T_{f}\left(z_{k}\right), r_{M}\left(z_{k}\right)\right), a_{k}=\psi_{1}\left(T_{f}\left(z_{k}\right), r_{M}\left(z_{k}\right)\right) m_{k}, k \geq 0$. Then $\psi_{2}\left(T_{f}\left(z_{k}\right), r_{M}\left(z_{k}\right)\right)=a_{k} m_{k}$. Since $T_{f}\left(z_{0}\right)<t_{2}\left(r_{M}\left(z_{0}\right)\right)$, we see that $a_{0} m_{0}=\psi_{2}\left(T_{f}\left(z_{0}\right), r_{M}\left(z_{0}\right)\right)<1$. Consequently, $a_{0}<1$ since $m_{0}>1$ and $m_{0}<1 / a_{0}$. By Lemmas 3.9, 4.7(a), 4.2, 4.3, we have

$$
\begin{gathered}
r_{M}\left(z_{1}\right) \leq m_{0} r_{M}\left(z_{0}\right), \\
T_{f}\left(z_{1}\right) \leq \psi_{1}\left(T_{f}\left(z_{0}\right), r_{M}\left(z_{0}\right)\right) T_{f}\left(z_{0}\right) \\
\leq \psi_{1}\left(T_{f}\left(z_{0}\right), r_{M}\left(z_{0}\right)\right) m_{0} T_{f}\left(z_{0}\right)=a_{0} T_{f}\left(z_{0}\right) .
\end{gathered}
$$

Note that $m_{0}=m\left(T_{f}\left(z_{0}\right), r_{M}\left(z_{0}\right)\right)<c$ by Lemma 4.7(b), that $m_{0}<1 / a_{0}$, and that $T_{f}\left(z_{0}\right)=1-u\left(r_{M}\left(z_{0}\right), m_{0}\right)$. So by (4.1), (4.2) and Lemmas 4.4, 4.5, 4.6, we have

$$
\begin{aligned}
m_{1}= & \frac{\left(1-T_{f}\left(z_{1}\right)\right)^{2}}{\left[\left(1+r_{M}\left(z_{1}\right)\right)\left(1-T_{f}\left(z_{1}\right)\right)^{2}-r_{M}\left(z_{1}\right)\right]^{2}} \\
\leq & \frac{\left(1-a_{0} T_{f}\left(z_{0}\right)\right)^{2}}{\left[\left(1+m_{0} r_{M}\left(z_{0}\right)\right)\left(1-a_{0} T_{f}\left(z_{0}\right)\right)^{2}-m_{0} r_{M}\left(z_{0}\right)\right]^{2}}=\left(g\left(r_{M}\left(z_{0}\right), m_{0}, a_{0}\right)\right)^{2} \\
\leq & \frac{1}{\left(1+a_{0} \sqrt{m_{0}}-a_{0} m_{0}\right)^{2}} \leq m_{0}, \\
a_{1} & =\frac{r_{M}\left(z_{1}\right) T_{f}\left(z_{1}\right) m_{1}^{2}}{2\left(1-T_{f}\left(z_{1}\right)\right)^{2}-1} \leq \frac{m_{0} r_{M}\left(z_{0}\right) \psi_{1}\left(T_{f}\left(z_{0}\right), r_{M}\left(z_{0}\right)\right) T_{f}\left(z_{0}\right) m_{0}^{2}}{2\left(1-T_{f}\left(z_{0}\right)\right)^{2}-1} \\
& =\psi_{1}\left(T_{f}\left(z_{0}\right), r_{M}\left(z_{0}\right)\right) m_{0} \frac{r_{M}\left(z_{0}\right) T_{f}\left(z_{0}\right) m_{0}^{2}}{2\left(1-T_{f}\left(z_{0}\right)\right)^{2}-1} \\
& =\left(\psi_{1}\left(T_{f}\left(z_{0}\right), r_{M}\left(z_{0}\right)\right) m_{0}\right)^{2}=a_{0}^{2} .
\end{aligned}
$$

Inductively, we have the following for all $n \geq 0$ :

$$
\begin{gathered}
r_{M}\left(z_{n+1}\right) \leq m_{n} r_{M}\left(z_{n}\right), \\
T_{f}\left(z_{n+1}\right) \leq \psi_{1}\left(T_{f}\left(z_{n}\right), r_{M}\left(z_{n}\right)\right) T_{f}\left(z_{n}\right) \\
\leq \psi_{1}\left(T_{f}\left(z_{n}\right), r_{M}\left(z_{n}\right)\right) m_{n} T_{f}\left(z_{n}\right)=a_{n} T_{f}\left(z_{n}\right), \\
m_{n+1} \leq m_{n}, \quad a_{n+1} \leq a_{n}^{2} .
\end{gathered}
$$


It follows that $T_{f}\left(z_{n+k}\right) \leq a_{k}^{2^{n}-1} T_{f}\left(z_{k}\right)$ for all $n \geq 0$ and $k \geq 0$. This can be proved by induction on $n$ for each $k$ as follows. Clearly, the inequality holds for $n=1$. Suppose that it holds for $n$; then

$$
T_{f}\left(z_{n+k+1}\right) \leq a_{n+k} T_{f}\left(z_{n+k}\right) \leq a_{k}^{2^{n}} a_{k}^{2^{n}-1} T_{f}\left(z_{k}\right)=a_{k}^{2^{n+1}-1} T_{f}\left(z_{k}\right) .
$$

So it also holds for $n+1$.

Theorem 2. If $T_{f}\left(z_{0}\right)<t_{2}\left(r_{M}\left(z_{0}\right)\right)$, then $z_{0}$ is an approximate zero of $f$ with respect to $M$, namely, there exist positive numbers $a_{0}, a_{1}, a_{2}, \ldots$ satisfying $a_{0}<1$ and $a_{k+1} \leq a_{k}^{2}$ for all $k \geq 0$ such that $\left|f\left(z_{n+k}\right)\right| \leq a_{k}^{2^{n}-1}\left|f\left(z_{k}\right)\right|$ for all $n \geq 0$ and $k \geq 0$, where $z_{n}=I_{f}^{n}\left(z_{0}\right)$.

Proof. Let $a_{0}, a_{1}, a_{2}, \ldots$ be as in Lemma 4.8; then we have $T_{f}\left(z_{n+1}\right) \leq$ $a_{n} T_{f}\left(z_{n}\right)$ for all $n \geq 0$ and $a_{k+1} \leq a_{k}^{2}$ for all $k \geq 0$. By Lemma 3.3,

$$
\left|f\left(z_{k+1}\right)\right| \leq \frac{r_{M}\left(z_{k}\right) T_{f}\left(z_{k}\right)}{1-T_{f}\left(z_{k}\right)}\left|f\left(z_{k}\right)\right|, \quad k \geq 0 .
$$

From this it follows that $\left|f\left(z_{n+k}\right)\right| \leq a_{k}^{2^{n}-1}\left|f\left(z_{k}\right)\right|$ for all $n \geq 0$ and $k \geq 0$. In fact, this is clearly valid for $n=0$. Now suppose that it is valid for $n$; then, by $(4.3)$,

$$
\begin{aligned}
& \left|f\left(z_{n+k+1}\right)\right| \leq \frac{r_{M}\left(z_{n+k}\right) T_{f}\left(z_{n+k}\right)}{1-T_{f}\left(z_{n+k}\right)}\left|f\left(z_{n+k}\right)\right| \\
& \quad \leq \frac{r_{M}\left(z_{n+k}\right) T_{f}\left(z_{n+k}\right)\left(1-T_{f}\left(z_{n+k}\right)\right)^{2} m_{n+k}\left|f\left(z_{n+k}\right)\right|}{\left[\left(1+r_{M}\left(z_{n+k}\right)\right)\left(1-T_{f}\left(z_{n+k}\right)\right)^{2}-r_{M}\left(z_{n+k}\right)\right]^{2}\left[2\left(1-T_{f}\left(z_{n+k}\right)\right)^{2}-1\right]} \\
& \quad=\psi_{1}\left(T_{f}\left(z_{n+k}\right), r_{M}\left(z_{n+k}\right)\right) m_{n+k}\left|f\left(z_{n+k}\right)\right| \\
& \quad=a_{n+k}\left|f\left(z_{n+k}\right)\right| \leq a_{k}^{2^{n}} a_{k}^{2^{n}-1}\left|f\left(z_{k}\right)\right|=a_{k}^{2^{n+1}-1}\left|f\left(z_{k}\right)\right| .
\end{aligned}
$$

The second inequality above makes use of the facts that $m_{n} \geq 1$ for all $n \geq 0$ and that

$$
\begin{aligned}
& \frac{1}{1-T_{f}\left(z_{n+k}\right)} \\
& \quad \leq \frac{\left(1-T_{f}\left(z_{n+k}\right)\right)^{2}}{\left[\left(1+r_{M}\left(z_{n+k}\right)\right)\left(1-T_{f}\left(z_{n+k}\right)\right)^{2}-r_{M}\left(z_{n+k}\right)\right]^{2}\left[2\left(1-T_{f}\left(z_{n+k}\right)\right)^{2}-1\right]} .
\end{aligned}
$$

The last inequality makes use of the fact that $a_{k+1} \leq a_{k}^{2}$ for all $k \geq 0$.

\section{NUMERICAL EXPERIMENTS AND DISCUSSION}

Our experimental work shows that the theoretical bounds given in the theorems are rather conservative, which echoes the findings by Curry and Van Vleck [2] about Smale's bound for Newton's method when applied to cubic polynomials. Nevertheless, these bounds provide a theoretical guarantee that a point satisfying the condition is an approximate zero of any given analytic function with respect to any given quadratically convergent iteration scheme $M$. 


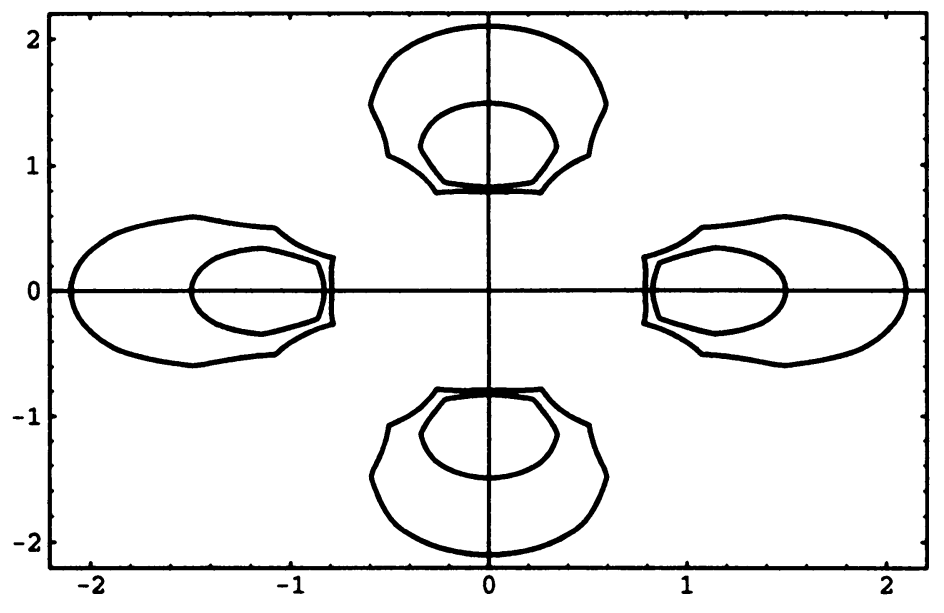

FIGURE 5.1

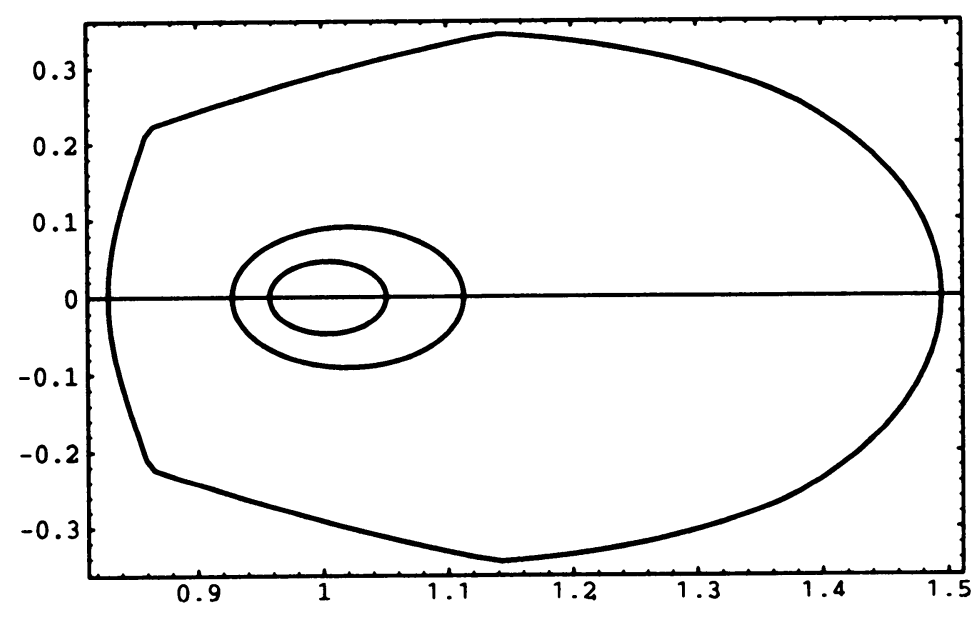

Figure 5.2

To illustrate our findings, we plot the true regions of fast convergence (i.e., true regions of approximate zeros) for the functions $f(z)=z^{4}-1$ and $f(z)=$ $e^{z}+z-1$ with respect to the Newton iteration, the iteration defined by $M(u, v)$ $=-u / v+u^{2} / v^{2}$, and the iteration defined by $M(u, v)=-u / v+u^{2}$. We also plot the regions of approximate zeros predicted by our conditions and that of Smale. The plots are made in the following way. For each region to be plotted, the boundary point of the region along each of the 128 evenly spaced radial lines from the zero is determined to a certain accuracy, and these 128 boundary points are then connected.

Figure 5.1 gives the true regions of approximate zeros with level $a_{0}=0.5$ (the inner contour) and $a_{0}=0.75$ (the outer contour) corresponding to the four zeros $1,-1, i,-i$ of the function $f(z)=z^{4}-1$ with respect to Newton iteration.

Figure 5.2 gives the true region of approximate zeros with level $a_{0}=0.5$ (the outer contour), the region of approximate zeros predicted by Smale's condition 


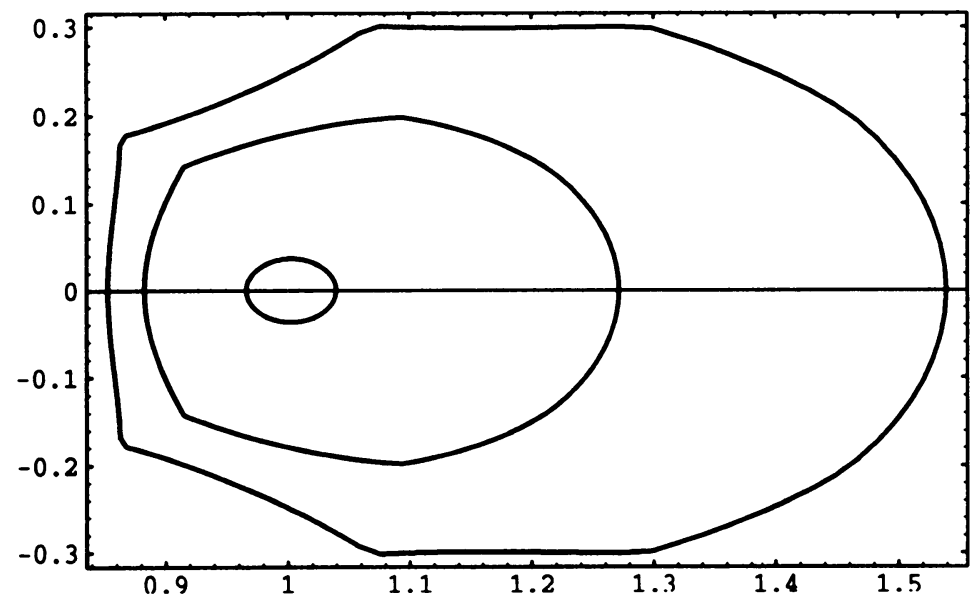

FIGURE 5.3

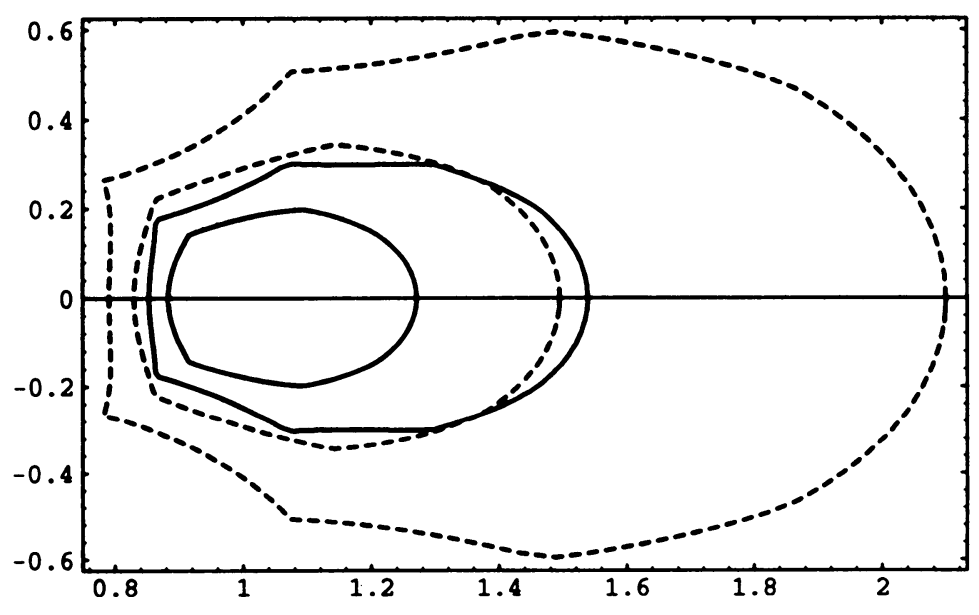

FIGURE 5.4

(the middle contour), and the region of approximate zeros predicted by our condition with $a_{0}=0.5$ (the inner contour) for the zero $z=1$ of the function $f(z)=z^{4}-1$ with respect to Newton iteration. When comparing the region of approximate zeros predicted by our condition with that by Smale's condition, one must bear in mind that our definition of an approximate zero is technically not quite the same as that of Smale and other people. So the comparison can only serve as a rough indication.

Figure 5.3 gives the true region of approximate zeros with level $a_{0}=0.75$ (the outer contour) and $a_{0}=0.5$ (the middle contour), and the region predicted by our condition (the inner contour) for the zero $z=1$ of the function $f(z)=$ $z^{4}-1$ with respect to the iteration defined by $M(u, v)=-u / v+u^{2} / v^{2}$.

Figure 5.4 gives the true regions of approximate zeros with level $a_{0}=0.5$ and $a_{0}=0.75$ of the function $f(z)=z^{4}-1$ around $z=1$ with respect 


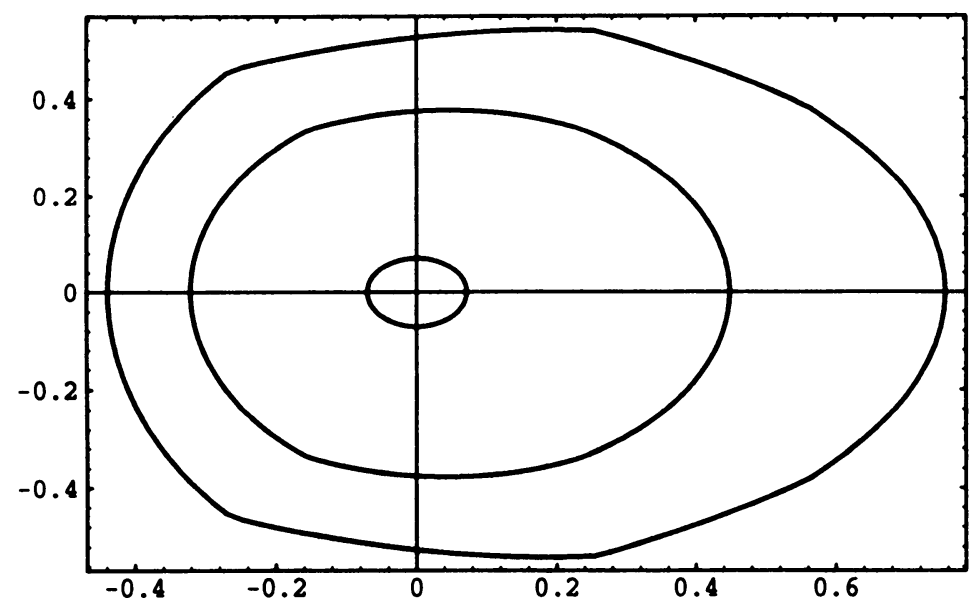

FIGURE 5.5

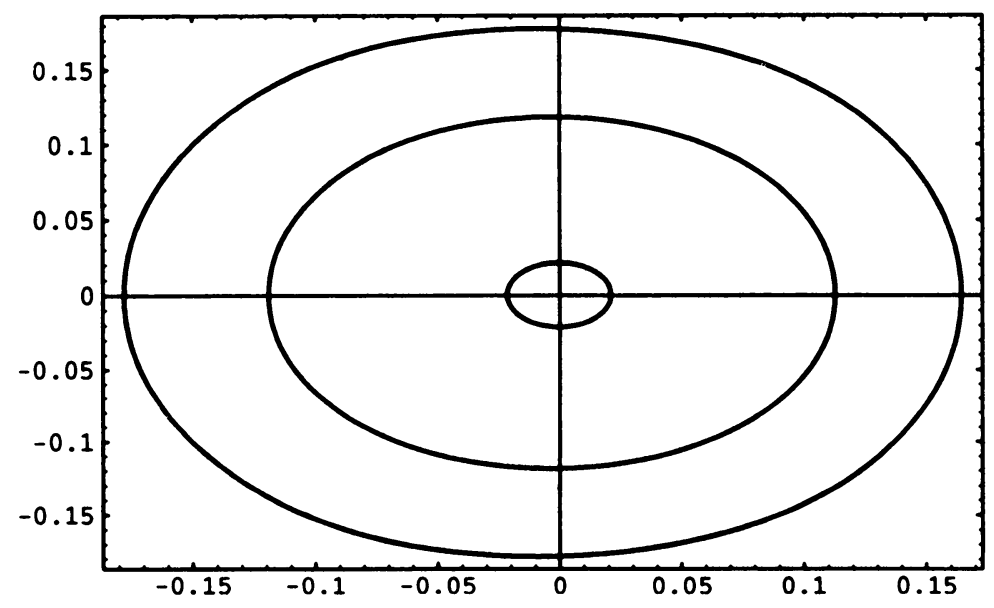

FIGURE 5.6

to both Newton iteration (the dashed contours) and the iteration defined by $M(u, v)=-u / v+u^{2} / v^{2}$.

Figure 5.5 gives the true region of approximate zeros with level $a_{0}=0.75$ (the outer contour) and $a_{0}=0.5$ (the middle contour), and the region predicted by our condition (the inner contour) for the zero $z=0$ of the function $f(z)=$ $e^{z}+z-1$ with respect to the iteration defined by $M(u, v)=-u / v+u^{2} / v^{2}$.

Figure 5.6 gives the true region of approximate zeros with level $a_{0}=0.75$ (the outer contour) and $a_{0}=0.5$ (the middle contour), and the region predicted by our condition (the inner contour) for the zero $z=0$ of the function $f(z)=$ $e^{z}+z-1$ with respect to the iteration defined by $M(u, v)=-u / v+u^{2}$.

As evidenced from these plots, the regions of approximate zeros predicted by our conditions are rather conservative; in fact, they seem to be completely contained in the true region of approximate zeros with level $a_{0}=0.5$. So it 
would be nice if the bounds could be improved. From Figure 5.4, it is seen that the regions of approximate zeros with respect to the iteration defined by $M(u, v)=-u / v+u^{2} / v^{2}$ are completely contained in the corresponding regions with respect to Newton iteration. Does this suggest any optimality of the Newton iteration compared to other quadratically convergent iterations in the sense of having the largest regions of approximate zeros?

\section{APPENDIX}

Proof of Lemma 3.9. Since $\psi_{1}\left(x, K_{M}\right)$ is increasing in $x$ on $\left[0,1-\sqrt{\frac{K_{M}}{1+K_{M}}}\right)$ and $\psi_{1}\left(t_{1}\left(K_{M}\right), K_{M}\right)=1$, it suffices to show that for all $K_{M} \geq 1$,

$$
\psi_{1}\left(\frac{1-\sqrt{\frac{K_{M}}{1+K_{M}}}}{2-\sqrt{\frac{K_{M}}{1+K_{M}}}}, K_{M}\right)>1 .
$$

Let $q=\sqrt{\frac{K_{M}}{1+K_{M}}}$; then $K_{M}=\frac{q^{2}}{1-q^{2}}$ and $\sqrt{2} / 2 \leq q<1$. Thus,

$$
\begin{aligned}
\psi_{1}\left(\frac{1-q}{2-q}, \frac{q^{2}}{1-q^{2}}\right) & =\frac{\left(\frac{q^{2}}{1-q^{2}}\right)\left(\frac{1-q}{2-q}\right)\left(1-\frac{1-q}{2-q}\right)^{2}}{\left[\left(1+\frac{q^{2}}{1-q^{2}}\right)\left(1-\frac{1-q}{2-q}\right)^{2}-\frac{q^{2}}{1-q^{2}}\right]^{2}\left[2\left(1-\frac{1-q}{2-q}\right)^{2}-1\right]} \\
& =\frac{q^{2}(1+q)^{3}}{\left(1-q^{2}\right)^{2}} \frac{1}{\left(1+2 q-q^{2}\right)^{2}} \frac{(2-q)^{3}}{\left[2-(2-q)^{2}\right]} \\
& =h_{1}(q) h_{2}(q) h_{3}(q),
\end{aligned}
$$

where

$$
h_{1}(x)=\frac{x^{2}(1+x)^{3}}{\left(1-x^{2}\right)^{2}}, \quad h_{2}(x)=\frac{1}{\left(1+2 x-x^{2}\right)^{2}}, \quad h_{3}(x)=\frac{(2-x)^{3}}{2-(2-x)^{2}} .
$$

It is easy to check that $h_{1}$ is positive and increasing on $\left[\frac{\sqrt{2}}{2}, 1\right)$, while $h_{2}$ and $h_{3}$ are positive and decreasing on $\left[\frac{\sqrt{2}}{2}, 1\right)$. So

$$
h_{1}(q) h_{2}(q) h_{3}(q) \geq h_{1}(\sqrt{2} / 2) h_{2}(1) h_{3}(1)=(1+\sqrt{2} / 2)^{3} / 2>1 .
$$

Proof of Lemma 4.4. The inequality to be proved is equivalent to

$$
(\sqrt{\mu}+\sqrt{\mu+4 r(1+r)}) \sqrt{\mu+r}>2 \sqrt{r}(1+r),
$$

where $\mu=1 / m$. By squaring both sides, we get the following equivalent inequality:

$$
\sqrt{\left(\mu^{2}+\mu r\right)\left(\mu^{2}+4 \mu r(1+r)+\mu r+4 r^{2}(1+r)\right)}>2(1-\mu) r(1+r)-\mu^{2}-\mu r .
$$


It is easy to see that the right-hand side of the inequality is nonpositive if

$$
\mu \geq \frac{\sqrt{4 r^{4}+12 r^{3}+17 r^{2}+8 r}-\left(2 r^{2}+3 r\right)}{2} .
$$

In this case, the inequality clearly holds. For the other case, squaring both sides again, we get the following equivalent inequality:

$$
\left(-\mu^{2}+3 \mu-1\right) r^{3}+\left(-\mu^{2}+6 \mu-2\right) r^{2}+\left(\mu^{2}+3 \mu-1\right) r+\mu^{2}>0
$$

Denote the left-hand side of the inequality by $h(r, \mu)$; then it can be checked that for $1 \geq \mu \geq(3-\sqrt{5}) / 2$, we have $h(1, \mu)>0, \partial h(1, \mu) / \partial r>0$, $\partial^{2} h(1, \mu) / \partial r^{2}>0$, and $\partial^{3} h(r, \mu) / \partial r^{3}=6\left(-\mu^{2}+3 \mu-1\right) \geq 0$. It follows that $h(r, \mu)>0$ for all $r \geq 1$ and $1 \geq \mu \geq(3-\sqrt{5}) / 2$. The lemma is proved by combining both cases and noting that $\mu=1 / m$ and that

$$
\frac{\sqrt{4 r^{4}+12 r^{3}+17 r^{2}+8 r}-\left(2 r^{2}+3 r\right)}{2}>\frac{3-\sqrt{5}}{2}, \quad r \geq 1 .
$$

Proof of Lemma 4.5. (a) We shall show that $\partial g(r, m, a) / \partial r \geq 0$. It can be calculated that

$$
\begin{aligned}
& \frac{\partial g(r, m, a)}{\partial r} \\
& \quad=\frac{m(1-a+a u)-a m r u^{\prime}-m(1-a+a u)^{3}-a(1+m r)(1-a+a u)^{2} u^{\prime}}{\left[(1+m r)(1-a+a u(r, m))^{2}-m r\right]^{2}}
\end{aligned}
$$

where $u=u(r, m)$ and $u^{\prime}=\partial u(r, m) / \partial r$. So we need to show that the numerator is nonnegative. To do that, we need the following facts about $u(r, m)$, which can be easily derived from the equation defining $u(r, m)$, namely, $u(r, m) /\left[(1+r)(u(r, m))^{2}-r\right]=\sqrt{m}$ :

(1) $0 \leq u(r, m) \leq 1$

(2) $\partial u(r, m) / \partial r=\sqrt{m}\left(1-u^{2}\right) /(2 \sqrt{m}(1+r) u-1)$;

(3) $\sqrt{m}(1+r) u-1=\sqrt{m} r / u$.

Using these facts, we see that the numerator is

$$
\begin{aligned}
m(1 & -a+a u)-a m r u^{\prime}-m(1-a+a u)^{3}-a(1+m r)(1-a+a u)^{2} u^{\prime} \\
& =m(1-a+a u)\left[1-(1-a+a u)^{2}\right]-a u^{\prime}\left[m r+(1+m r)(1-a+a u)^{2}\right] \\
& =\frac{a \sqrt{m}(1-u)}{2 \sqrt{m}(1+r) u-1} H(r, m, a),
\end{aligned}
$$

where 


$$
\begin{aligned}
H(r, & m, a)=\sqrt{m}(1-a+a u)(2-a+a u)[2 \sqrt{m}(1+r) u-1] \\
& -(1+u)\left[m r+(1+m r)(1-a+a u)^{2}\right] \\
= & m(1+r)(1-a+a u)^{2} u+\sqrt{m}(1-a+a u)^{2}[\sqrt{m}(1+r) u-1] \\
& +\sqrt{m}(1-a+a u)+2 \sqrt{m}(1-a+a u)[\sqrt{m}(1+r) u-1] \\
& -(1+u)\left[m r+(1+m r)(1-a+a u)^{2}\right] \\
= & m(1+r)(1-a+a u)^{2} u+\frac{\sqrt{m} \sqrt{m} r(1-a+a u)^{2}}{u}+\sqrt{m}(1-a+a u) \\
& +\frac{2 \sqrt{m} \sqrt{m} r(1-a+a u)}{u}-(1+u)\left[m r+(1+m r)(1-a+a u)^{2}\right] \\
= & m(1+r)(1-a+a u)^{2} u+\frac{m r(1-a+a u)^{2}}{u}+\sqrt{m}(1-a+a u) \\
& +\frac{2 m r(1-a+a u)}{u}-m r-(1-a+a u)^{2} \\
& -m r(1-a+a u)^{2}-m r u-(1+m r)(1-a+a u)^{2} u \\
= & (m-1)(1-a+a u)^{2} u+\frac{m r(1-a+a u)^{2}(1-u)}{u} \\
& +(1-a+a u)[(\sqrt{m}-1)+a(1-u)]+\frac{m r(1-a)(1-u)}{u} \\
& +\frac{m r(1-a+u)(1-u)}{u} \geq 0,
\end{aligned}
$$

since every term is nonnegative.

(b) It is easy to see that

$$
u(r, m)=\frac{1+\sqrt{1+4 m r(1+r)}}{2 \sqrt{m}(1+r)} \rightarrow 1 \quad(r \rightarrow \infty)
$$

So the numerator $1-a+a u \rightarrow 1(r \rightarrow \infty)$. For the denominator, we see that as $r \rightarrow \infty$,

$$
\begin{aligned}
& (1+m r)(1-a+a u)^{2}-m r \\
& \quad=(1-a+a u)^{2}+a m r(u-1)(2-a+a u) \rightarrow 1+2 a m \lim _{r \rightarrow \infty} r(u-1) .
\end{aligned}
$$

To calculate $\lim _{r \rightarrow \infty} r(u-1)$, we note that

$$
\begin{aligned}
r(u-1) & =\frac{1}{2 \sqrt{m}} \frac{r}{1+r}\left(1+\frac{1-4 m-4 m r}{\sqrt{1+4 m r(1+r)}+2 \sqrt{m}(1+r)}\right) \\
& \rightarrow \frac{1-\sqrt{m}}{2 \sqrt{m}} \quad(r \rightarrow \infty) .
\end{aligned}
$$

So $\lim _{r \rightarrow \infty} g(r, m, a)=1 /(1+a \sqrt{m}-a m)$.

(c) Part (c) follows immediately from parts (a) and (b).

Proof of Lemma 4.6. (a) straightforward. 
(b) Since $1+a \sqrt{m}-a m>0$ by part (a), the inequality is equivalent to $1 \leq \sqrt{m}(1+a \sqrt{m}-a m)$ or $a m \sqrt{m}-a m-\sqrt{m}+1=(\sqrt{m}-1)(a m-1) \leq 0$.

Proof of Lemma 4.7. (a) Since $m(x, r)>1$ for $x>0$, we have $\psi_{2}(x, r)=$ $\psi_{1}(x, r)(m(x, r))^{2}>\psi_{1}(x, r)$. So $t_{2}(r)<t_{1}(r)$ by the definitions of $t_{1}(r)$ and $t_{2}(r)$ and the fact that both $\psi_{1}(x, r)$ and $\psi_{2}(x, r)$ are increasing in $x$ on $[0,1-\sqrt{r /(1+r)})$.

(b) Notice that $m(x, r)$ is increasing in $x$ on $[0,1-\sqrt{r /(1+r)})$ and maps $[0,1-\sqrt{r /(1+r)})$ onto $[1, \infty)$. Let $s(r)$ be the unique number in $[0,1-$ $\sqrt{r /(1+r)})$ such that $m(s(r), r)=c$. We shall show that $\psi_{2}(s(r), r)>1$ for all $r \geq 1$, from which it follows that $t_{2}(r)<s(r)$ for all $r \geq 1$, and consequently, $m\left(t_{2}(r), r\right)<c$ for all $r \geq 1$. Since $0<s(r)<1$, we have $2(1-s(r))^{2}-1 \leq 1$ for all $r \geq 1$. So, noting that $s(r)=1-u(r, c)$, we have for all $r \geq 1$

$$
\begin{aligned}
\psi_{2}(s(r), r) & =\frac{s(r) r[m(s(r), r)]^{3}}{2(1-s(r))^{2}-1} \geq c^{3} r s(r)=c^{3} r(1-u(r, c)) \\
& =c^{3} r\left(1-\frac{1+\sqrt{1+4 c r(1+r)}}{2 \sqrt{c}(1+r)}\right) \\
& =\frac{c^{2} \sqrt{c}}{2}\left(\frac{r}{1+r}\right)\left(\frac{4 c-1+4 c r}{2 \sqrt{c}(1+r)+\sqrt{1+4 c r(1+r)}}-1\right) \\
& \geq \frac{c^{2} \sqrt{c}}{4}\left(\lim _{r \rightarrow \infty} \frac{4 c-1+4 c r}{2 \sqrt{c}(1+r)+\sqrt{1+4 c r(1+r)}-1)}\right. \\
& =\frac{c^{2} \sqrt{c}}{4}(\sqrt{c}-1)>1 .
\end{aligned}
$$

In the above, the facts that $r /(1+r) \geq 1 / 2$ for all $r \geq 1$ and that

$$
\frac{4 c-1+4 c r}{2 \sqrt{c}(1+r)+\sqrt{1+4 c r(1+r)}}
$$

is decreasing in $r$ on $[1, \infty)$ are used.

\section{BIBLIOGRAPHY}

1. J.H. Curry, On zero finding methods of higher order from data at one point, J. Complexity 5 (1989), 219-237.

2. J.H. Curry and E.S. Van Vleck, On the theory and computation of approximate zeros, preprint.

3. M. Kim, On approximate zeros and rootfinding algorithms for a complex polynomial, Math. Comp. 51 (1988), 707-719.

4. M. Shub and S. Smale, Computational complexity: on the geometry of polynomials and a theory of costs. I, Ann. Sci. École Norm. Sup. (4) 18 (1985), 107-142.

5. Computational complexity: on the geometry of polynomials and a theory of costs.II, SIAM J. Comput. 15 (1986), 145-161.

6. S. Smale, The fundamental theorem of algebra and complexity theory, Bull. Amer. Math. Soc. (N.S.) 4 (1981), 1-36. 
7. - On the efficiency of algorithms of analysis, Bull. Amer. Math. Soc. (N.S.) 13 (1985), 87-121.

8. __ Newton's method estimates from data at one point, The Merging of Disciplines: New Directions in Pure, Applied, and Computational Mathematics (Richard E. Ewing, Kenneth I. Gross, and Clyde F. Martin, eds.), Springer-Verlag, 1986, pp. 185-196.

9. _ Algorithms for solving equations, Proc. Internat. Congr. Math. (Berkeley, CA, 1986), Amer. Math. Soc., Providence, RI, 1987, pp. 172-195.

10. W.C. Rheinboldt, On a theorem of S. Smale about Newton's method for analytic mappings, Appl. Math. Lett. 1 (1988), 69-72.

Department of Mathematics and Computer Science, Gustavus Adolphus College, SAint Peter, Minnesota 56082

E-mail address: chenegac.edu 